\title{
Novel interventional radiological management for lymphatic leakages after gynecologic surgery: lymphangiography and embolization
}

\author{
Saebeom Hur, Hoyong Jun, Yoon Soo Jeong \\ Department of Radiology, Seoul National University Hospital, Seoul, Korea \\ Contributions: (I) Conception and design: S Hur; (II) Administrative support: S Hur; (III) Provision of study materials or patients: H Jun; (IV) \\ Collection and assembly of data: YS Jeong; (V) Data analysis and interpretation: H Jun, YS Jeong; (VI) Manuscript writing: All authors; (VII) Final \\ approval of manuscript: All authors. \\ Correspondence to: Saebeom Hur, MD. Department of Radiology, Seoul National University College of Medicine, Seoul National University Hospital, \\ 101 Daehak-ro, Jongro-gu, Seoul 110-744, Korea. Email: saebeom.hur@snu.ac.kr; hurz21@gmail.com.
}

\begin{abstract}
Post-operative lymphatic leakage is a common complication of a radical gynecologic surgery involving aggressive lymph node dissection. Its manifestation varies from asymptomatic lymphoceles to lifethreatening chylous ascites. In the past, nuclear medicine lymphoscintigraphy was the sole imaging modality for the confirmation of the leakage, of which application is limited due to its poor spatial resolution. While a conservative treatment with percutaneous drainage was the mainstream treatment method, surgical exploration was the last resort for the recalcitrant leakages. Recently, there have been a series of innovations in the field of interventional radiology, including intranodal Lipiodol ${ }^{\circledR}$ lymphangiography, dynamic magnetic resonance (MR) lymphangiography, lymphatic embolization, and mesenteric lymph node lymphangiography. Intranodal Lipiodol ${ }^{\circledR}$ lymphangiography provides very reliable and secure access to the lymphatic system, while requiring only fundamental skills and equipment available to all interventional radiologists. Besides, Lipiodol $^{\circledR}$ is being received a spotlight for its potential therapeutic effects on refractory lymphatic leakage. Dynamic MR lymphangiography plays a vital role in the diagnosis and management of non-traumatic lymphatic diseases, as well as in the post-operative lymphatic leakage with its superior contrast, spatial and temporal resolution. Lymphatic embolization is a technique of using N-BCA glue, a liquid embolic agent, for lymphatic leakages. It can be further described as lymphopseudoaneurysm (LPA) embolization or lymph node embolization, according to which structure is being embolized. Lymphatic embolization opened a new realm of possibilities in the field of interventional lymphology, resulting in the development of treatment approaches for chylous ascites and lymphoceles. Mesenteric lymph node lymphangiography offers the opacification of the upstream mesenteric chylous lymphatic system and can detect lymphatic leakage from the intestinal trunk or the more proximal parts of the mesenteric lymphatic system. With the advent of these latest interventional radiological techniques, more comprehensive approaches to the management of recalcitrant post-operative lymphatic leakages have been enabled.
\end{abstract}

Keywords: Lymphocele; lymphatic leakage; chylous; lymphangiography; embolization

Submitted Feb 18, 2020. Accepted for publication Aug 07, 2020.

doi: $10.21037 /$ gs-2019-ursoc-10

View this article at: http://dx.doi.org/10.21037/gs-2019-ursoc-10

\section{Introduction of post-operative lymphatic leakage}

The main function of the lymphatic system is to collect and return the interstitial fluid to the venous system (1).
The lymphatic system also plays an important role in the transportation of large molecules, such as absorbed lipids. The lymphatic system begins with the lymphatic capillaries, 
which drain into collecting lymphatic vessels and lymph nodes (2). The complex network of interlacing lymphatic vessels and lymph nodes then distributes along the major vascular structures.

The lymphatic system consists of soft tissue, intestinal, and liver parts, which finally coalesce together at the level of the cisterna chyli. The terminal drainage of lymphatic fluid occurs via the veins through lympho-venous connections. Of these connections, the most important is located between the thoracic duct and the junction of the left internal jugular veins and the left subclavian vein (3). Iatrogenic injury to these lymphatic structures may cause post-operative lymphatic leakage that shows distinct clinical features according to the involved anatomy.

The lymph system is also important for the management of gynecologic malignancies, which require pelvic and paraaortic lymph node dissection as an indispensable part of the surgery (4). Compared to blood vessels, the lymphatic vessels are small, and the leakage is slow and transparent, so lymphatic leakage is more difficult to notice during surgery. In addition, the lymph does not undergo any spontaneous coagulation action. Nevertheless, this leakage spontaneously resolves without the development of symptoms in the majority of cases. However, when the leakage persists, as it does in some patients, lymphocele (lymphorrhea confined to the cavity), cutaneous lymphatic leakage, or lymphatic ascites might develop.

The majority of lymphoceles are asymptomatic and require no further treatment. A recent prospective study on 800 consecutive patients reported an incidence of asymptomatic and symptomatic lymphoceles of $20.2 \%$ and $5.8 \%$, respectively (5). If symptomatic, a lymphocele may result in significant post-operative morbidity and may even delay further cancer treatment. The symptoms can include pain, hydronephrosis, urinary urgency, or thrombosis, which are caused by the compression of adjacent structures (ureters, urinary bladder, rectum, or large vessels). All lymphoceles, regardless of the symptoms, can also be a focus of infection.

If the drained fluid shows a milky or creamy appearance, the possibility of chyle leakage should be considered. Since chyle originates solely from the small bowel, the chylous leakage strongly suggests an injury to the mesenteric lymphatic system, intestinal trunk, cisterna chyli, or thoracic duct, or to the retroperitoneal lymphatic network, which is close to the aforementioned structures. The presentation of chylous leakage as a retroperitoneal chylous lymphocele, chylous ascites, or chylothorax depends on the location and extent of the lymphatic injury and the integrity of the adjacent retroperitoneal structures. Chylous leakage tends to cause more severe morbidity in patients because it contains higher concentrations of nutrients, and the amount leaked is usually more excessive than occurs with non-chylous leakage. Many surgical series have shown that patients with chylous ascites have increased morbidity, reduced survival, and prolonged hospital stays (6).

\section{Diagnosis}

\section{Conventional imaging studies: computed tomography (CT), magnetic resonance (MR), or ultrasound (US)}

Because of the small size and complexity of the lymphatic vessels, observation usually requires introduction of a contrast agent, whereas lymph nodes exceeding a few millimeters can be seen in general radiologic imaging modalities, such as CT, MR, or US imaging. Recently, coronal heavily T2-weighted imaging using 3.0-T MR imaging (MRI) has been used to visualize components of the central lymphatic system, such as cisterna chyli or thoracic duct, without injection of contrast agent (7). However, the clinical usefulness of imaging is limited by the fact that it cannot visualize either non-dilated structures or the lymphatic flow. The primary role of these conventional radiology modalities in post-operative lymphatic leakage is limited to the evaluation of the fluid collection and its relationship with the symptoms.

\section{Nuclear medicine lymphoscintigraphy}

Lymphoscintigraphy can visualize the physiological lymphatic flow using radioactive materials (radiotracers) that are typically injected subcutaneously and enter the lymphatic system. The greatest advantages of lymphoscintigraphy over other modalities are its simplicity and reliability, as introducing the radiotracer does not require specialized skills or equipment. Radiotracers also show the same flow characteristics as lymphatic fluid because they have the same water-soluble nature, unlike the oil-soluble materials like Lipiodol ${ }^{\circledast}$ that are used for lymphangiography (8). However, lymphoscintigraphy has clear limitations in terms of time and spatial resolution for localizing an exact leakage point (Figure 1).

\section{$X$-ray lymphangiography}

Traditionally, the term lymphangiography or lymphography 

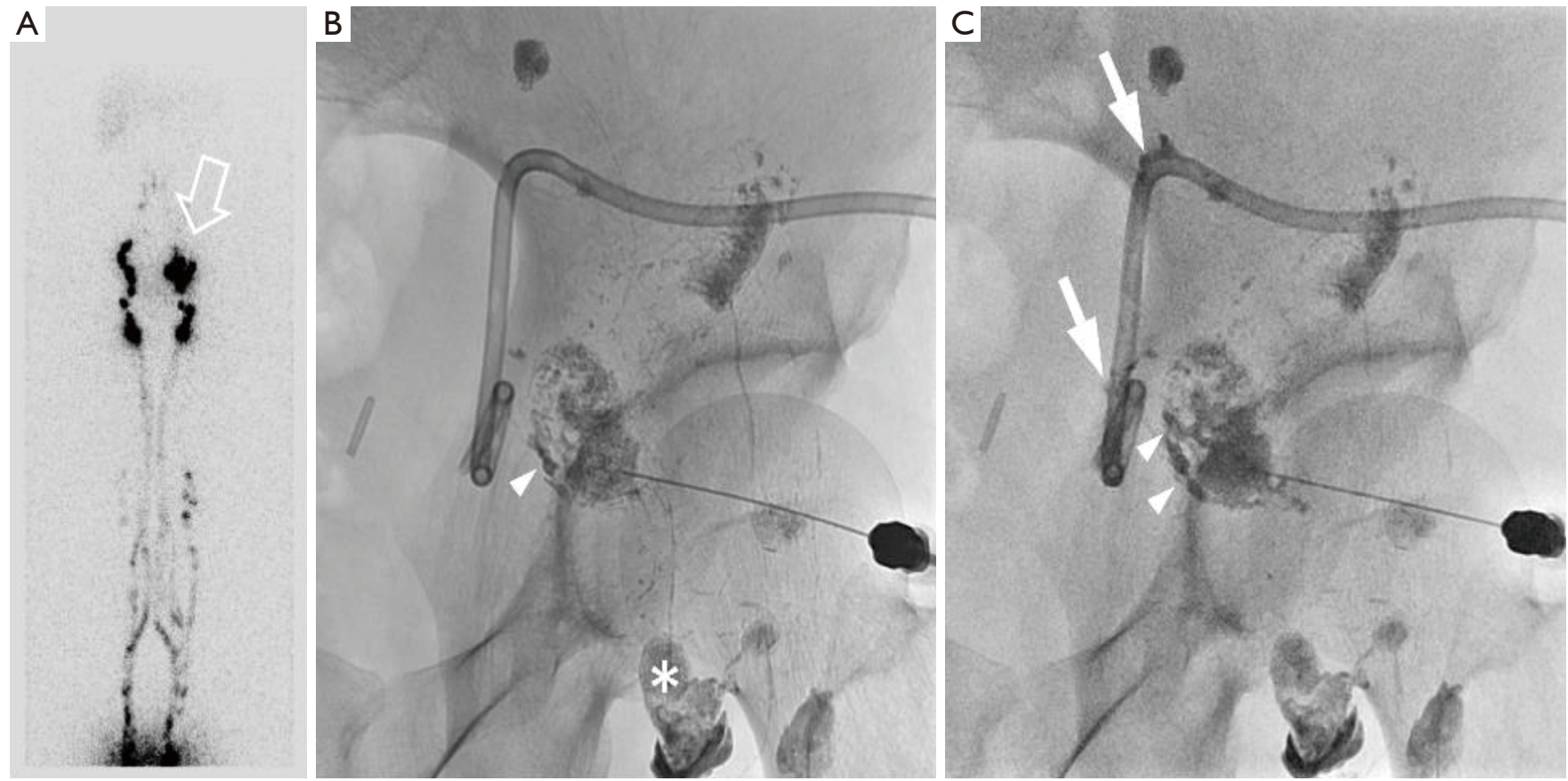

Figure 1 A 43-year-old female with a symptomatic pelvic lymphocele after pelvic lymph node dissection for ovarian cancer. (A) Lymphoscintigraphy showed an increased radioactivity of the left pelvic lymphocele (open arrow); (B) an intranodal lymphangiography through an inguinal lymph node (asterisk) showed an extravasation of Lipiodol ${ }^{\circledR}$ (arrowhead) from a medial external iliac lymph node, which was punctured with another needle under fluoroscopic guidance; (C) after embolization of the medial external iliac lymph node using diluted glue, Lipiodol ${ }^{\circledR}$ and glue leaked into the lymphocele and collected around the drainage catheter (white arrows).

referred to the X-ray imaging of the lymphatic system after opacification with Lipiodol ${ }^{\circledR}$. The use of Lipiodol ${ }^{\circledR}$ as the contrast agent for lymphangiography was established in the 1960s, as it is retained in the lymphatic system, unlike iodinated water-soluble contrast media that diffuse out of the lymphatics rather quickly. Lymphangiography had initially been used for the evaluation of lymph nodes in patients with lymphoma or suspected lymph node metastases. However, it essentially became forgotten after the development of cross-sectional imaging that could quickly evaluate the status of lymph nodes (9). Transpedal lymphangiography, which requires direct puncturing of the fine lymphatic vessel in the dorsum of the foot with a 30-gauge needle after surgical exposure, has been the mainstay of the direct lymphangiography for a long time. Its main problem is that it requires special surgical skills and experience by the operator for its success. The examination also takes a very long time to complete because of the fragility of the lymphatic vessel, which limits the speed of injection of Lipiodol ${ }^{\circledR}$, and because of the long course through the leg lymphatics that must be passed before the injected Lipiodol ${ }^{\circledR}$ reaches the body trunk.

Intranodal lymphangiography offers an alternative means for direct lymphatic access. Since the first description of US-guided intranodal lymphangiography in 2011 (10), it has virtually replaced transpedal lymphangiography for most indications. The advantage of intranodal lymphangiography is that it provides very reliable and secure access to the lymphatic system, while requiring only fundamental skills and equipment available to all interventional radiologists, namely the US-guided puncture of centimeter-sized structures. The time to obtain adequate imaging of the body lymphatic system has been dramatically reduced because the robust lymph node parenchyma can accept a higher rate of contrast agent injection, and the long lymphatic vessels of the leg are bypassed.

\section{Dynamic MR lymphangiography}

Dynamic MR lymphangiography, first introduced in 2014, now plays a vital role in the diagnosis and management of non-traumatic lymphatic diseases, as well as in the 

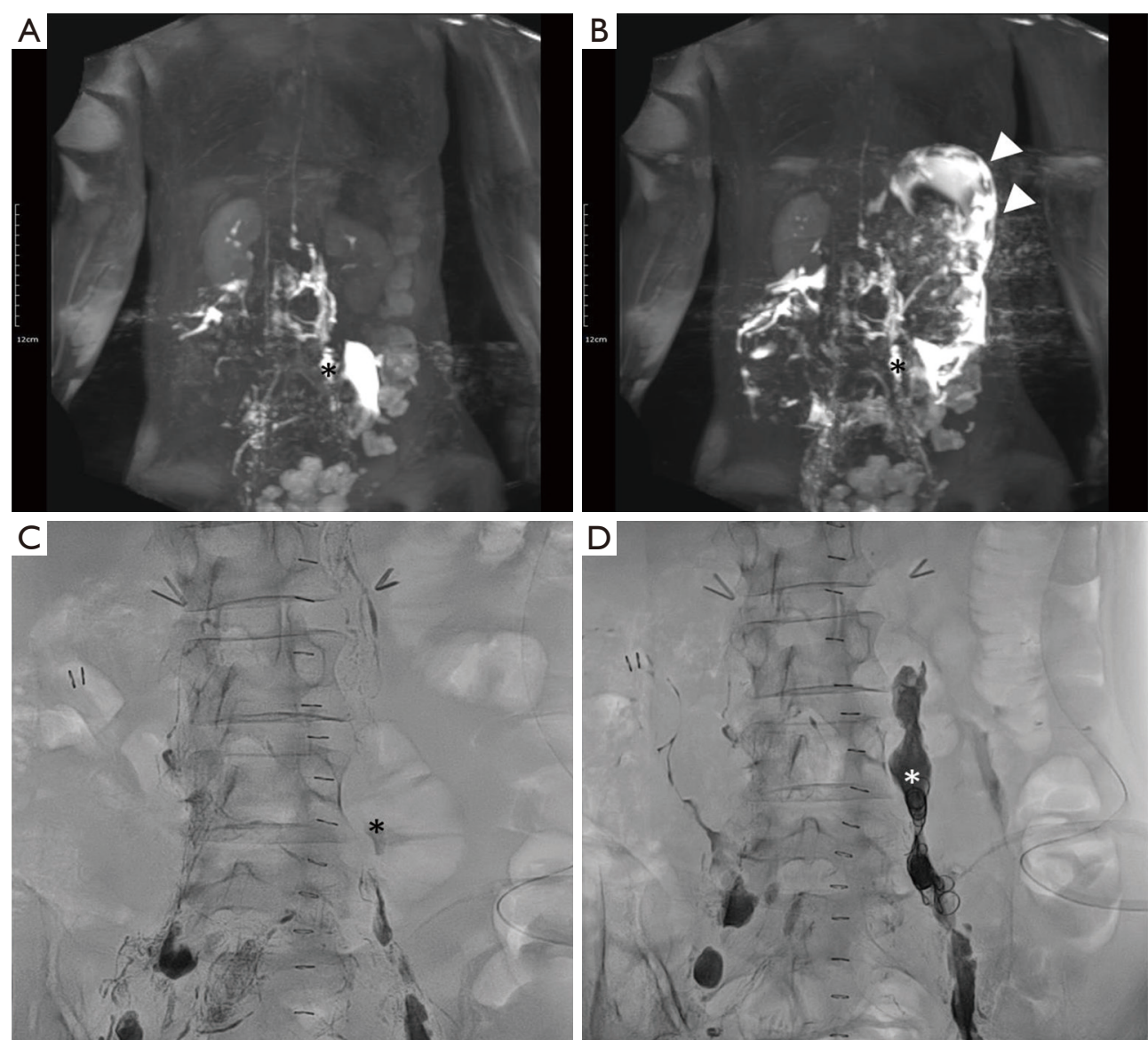

Figure 2 A 54-year-old female with chylous ascites after para-aortic lymph node dissection for ovarian cancer. (A,B) Dynamic contrastenhanced MR lymphangiography showed the collection of the extravasated contrast agent in the retroperitoneal lymphocele (asterisk) and its leakage into the peritoneal space (arrowheads), thereby confirming that the lymphatic leakage had caused the post-operative ascites; (C) Lipiodol $^{\circledR}$ extravasated into the lymphocele, which was not fully visualized in the intranodal lymphangiography (asterisk); (D) the lymphocele (asterisk) was embolized using glue and coils, based on the confirmative MRI findings. MR, magnetic resonance; MRI, MR imaging.

post-operative lymphatic leakage. This procedure can provide lymphatic flow information as well as anatomical information for the full extent of the affected area $(11,12)$ (Figure 2).

\section{Interventional management}

\section{Percutaneous drainage and medical therapy}

Only symptomatic lymphoceles should be treated. The first step is to ensure adequate drainage by placing a drainage catheter into the body cavity or the lymphocele, except for cases of cutaneous lymphorrhea (13). This drainage relieves the compression of the adjacent organs caused by the leakage, removes the potential or active infection focus, and promotes the spontaneous healing of the damaged lymphatic vessels or lymph nodes. Daily monitoring of the drainage amount also enables an objective evaluation of the response to the treatment. A malfunctioning drainage tube should be changed as soon as reasonably possible. A catheter with a diameter of at least $8.5 \mathrm{Fr}$ is recommended to prevent frequent clogging (14).

Dietary modifications, with total parenteral nutrition or a fat-free diet, and medical therapies, including octreotide, might help control the leakage of chylous lymphatic fluid by reducing the chylous flow (15). Octreotide is a synthetic somatostatin analog that inhibits lymph fluid excretion. It causes mild vasoconstriction of both the arterial and lymphatic vasculature of the gut, thereby lowering the hepatic venous flow and minimizing 
lymphatic fluid production, which indirectly lessens chylous flow (16).

\section{Sclerotherapy}

Sclerotherapy is performed by filling the closed lymphocele with a sclerosing agent through a pre-placed drainage catheter. The proposed mechanism of action is irritation of the nonepithelial wall, which results in local inflammation and fibrosis of the lymphatic channels (17).

Since the original description in 1982, several agents (e.g., ethanol, bleomycin, iodine, doxycycline, talc, and sodium tetradecyl sulfate) have been described for lymphocele sclerotherapy, with a reported success rate of around $80 \%$. Ethanol sclerotherapy is safe, effective, and less invasive than surgical marsupialization, with reported success rates of $70-100 \%$, a mean duration of catheter drainage of $17-$ 22 days, and complication rates of $6-20 \%$, according to several retrospective studies (14).

Technical simplicity is the great strength of this procedure, as it frees the procedure from the experience and skills of the operator. However, a requirement for repeated procedures and, hence, a prolonged treatment period is the downside of the sclerotherapy. Its application can also be limited by continuity with the peritoneal cavity. It is also known to be less effective when the volume of the lymphocele is large, and the daily output is high (14).

\section{Thoracic duct embolization}

Iatrogenic injury of the thoracic duct can cause a highoutput chylothorax, which requires intervention. Thoracic duct embolization for traumatic chylothorax has been gaining acceptance and use as first-line therapy for chylous leaks since its first introduction (18). Even though some physicians still hesitate at the idea of needle passage through the vital organs in the abdomen, safety information about this procedure is continuously being accumulated (19). The technical success rate of thoracic duct embolization was reported as around $50-80 \%$ in the historical literature (20). However, it is currently believed to be closer to $90 \%$ in experienced centers with the advancement of lymphangiography and other interventional techniques. Even though the thoracic duct is less likely to be injured during the surgery for gynecologic malignancy, if injury occurs, thoracic duct embolization can be a good treatment option over invasive surgical thoracic duct ligation.

\section{Therapeutic effect of Lipiodol ${ }^{\circledR}$}

The original intention of Lipiodol ${ }^{\circledR}$ lymphangiography was to visualize leakage points or the cisterna chyli during thoracic duct embolization; however, it has since received a spotlight for its potential therapeutic effects on refractory lymphatic leakage (21-26). Lipiodol $^{\circledR}$-induced selective blockage of the pathological lymph ducts and its sterile inflammatory reactions are believed to lead to scarring; hence, lymphatic leakage can undergo healing within a few days or weeks (9). However, a significant proportion of patients do not show a response, especially those with higher drainage volume who are most in need of definitive treatment.

\section{Lymphatic embolization}

Since the therapeutic efficacy of Lipiodol ${ }^{\circledR}$ itself is limited for the control of intractable lymphatic leakage after lymph node dissection, adjunctive embolization methods have been attempted, and some are reported to show promising outcomes (27).

\section{Lymphopseudoaneurysm (LPA) embolization}

"LPA" was defined as small extravasated lymphatic fluid collections contained by the surrounding tissue before draining into larger spaces, such as the peritoneum or a lymphocele (27). The concept of LPA embolization has been introduced in several case reports and case series (28-30). Although the terminologies differed in each paper, the method of LPA embolization with glue using a percutaneous approach was the same. While most of the successful treatments were obtained for chylous leakage in retroperitoneal lymphoceles, the same did not hold for pelvic lymphoceles. In one study, eight cases of LPA embolization were performed, but with only a $50 \%$ success rate (27). The success rate of this method can be limited by the fact that the glue injected into a pseudoaneurysm in retrograde fashion cannot overflow to cast the damaged lymphatic vessels many times. In those cases, the treatment success depends on the balance between lymphatic pressure from the damaged channel and resistance of the LPA filled with the glue cast. Chylous lymphoceles in the retroperitoneum tend to have a lower lymphatic pressure than that of the pelvic lymphocele if the drainage route through the thoracic duct to the venous system is maintained (Figure 2). 

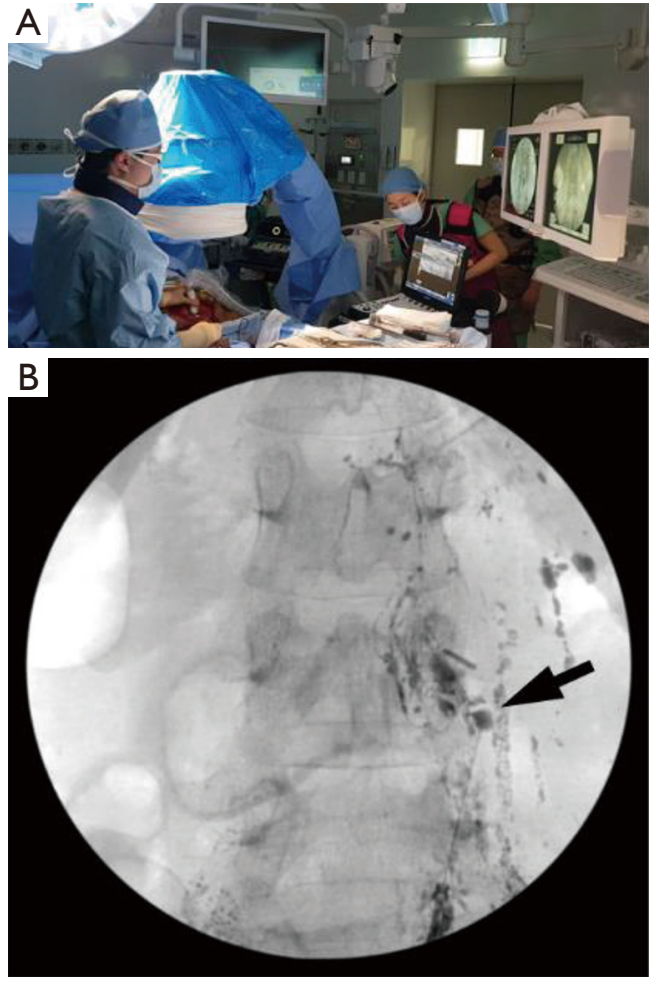

Figure 3 A 22-year-old female with non-traumatic chylous ascites. (A) Mesenteric intranodal lymphangiography required open laparotomy to expose the mesentery for the US-guided access to a small mesenteric lymph node; (B) Lipiodol ${ }^{\circledR}$ was carefully injected into the punctured lymph node (arrow) to evaluate the mesenteric lymphatic system. US, ultrasound.

\section{Lymph node embolization (interstitial embolization)}

Liquid embolic agents, such as NBCA glue, can be injected from a lymph node upstream of the leakage point in an antegrade fashion, and this glue can potentially cast the damaged lymphatic channels securely (Figure 1). Lymph node embolization can be performed in the same way as intranodal lymphangiography. The NBCA glue and Lipiodol ${ }^{\circledR}$ mixture can be injected into the lymph node just like Lipiodol ${ }^{\circledR}$ alone until the polymerization reaction occurs. The ratio of glue to Lipiodol $^{\circledR}$ should be adjusted according to the extent of the target embolization; this ratio can range from 1:3 to $1: 9$ (27).

\section{Mesenteric lymphangiography}

Chylous lymphatic fluid solely formed in the small bowel travels through the mesenteric lymphatic system to join the cisterna chyli via its intestinal trunk. Because contrast medium injected through inguinal or retroperitoneal lymph nodes usually does not move against the flow direction of the chylous lymphatic fluid, the intestinal trunk and its upstream mesenteric lymphatic system are rarely visualized in any lymphatic examination. Mesenteric lymph node lymphangiography offers the opacification of the upstream mesenteric chylous lymphatic system and can detect lymphatic leakage from the intestinal trunk or the more proximal parts of the mesenteric lymphatic system (31). If this approach detects a mesenteric lymphatic leakage site, it can be embolized or surgically treated afterward (9). The disadvantage of mesenteric lymphangiography is that it requires exploratory laparotomy to expose the mesenteric lymph nodes for the US-guided puncture (Figure 3).

\section{Conclusions}

The application of lymphangiography and various lymphatic interventional techniques is still in its infancy; therefore, robust data on the subject are scarce. Furthermore, the current literature is limited to case reports and retrospective cohorts that do not allow drawing of definitive conclusions. However, the reports so far have been consistent in demonstrating the potential of lymphatic interventions, particularly as an alternative bailout measure for lesions that are refractory to conservative treatment, sclerotherapy, or simple lymphangiography $(19,27,32,33)$. Initial treatment failure may occur on occasion, owing to pervasive damage to the lymph nodes and lymphatic vessels that form this complex network. Additional sessions of embolization or combined sclerotherapy may also be performed to enhance the treatment effect. One potential advantage of embolization over sclerotherapy is that embolization is not contraindicated for immature or ruptured lymphoceles that freely communicate with the peritoneal space. Regardless of all the limitations mentioned above, lymphangiography and various lymphatic interventional techniques are expected to have a broader role in the management of lymphatic leakage after gynecologic surgery.

\section{Acknowledgments}

Funding: This article was supported by the Basic Science Research Program of the National Research Foundation of Korea (Grant No. NRF-2018R1C1B6007875), which is funded by the Ministry of Science, ICT and Future Planning. 


\section{Footnote}

Provenance and Peer Review: This article was commissioned by the Guest Editors (Sang Yoon Park, Jae Weon Kim) for the series "Ultra-Radical Surgery in Ovarian Cancer: Surgical Techniques for Gynecologic Oncologist" published in Gland Surgery. The article was sent for external peer review organized by the Guest Editors and the editorial office.

Conflicts of Interest: All authors have completed the ICMJE uniform disclosure form (available at http://dx. doi. org/10. 21037/gs-2019-ursoc-10). The series "UltraRadical Surgery in Ovarian Cancer: Surgical Techniques for Gynecologic Oncologist" was commissioned by the editorial office without any funding or sponsorship. The authors have no other conflicts of interest to declare.

Ethical Statement: The authors are accountable for all aspects of the work in ensuring that questions related to the accuracy or integrity of any part of the work are appropriately investigated and resolved.

Open Access Statement: This is an Open Access article distributed in accordance with the Creative Commons Attribution-NonCommercial-NoDerivs 4.0 International License (CC BY-NC-ND 4.0), which permits the noncommercial replication and distribution of the article with the strict proviso that no changes or edits are made and the original work is properly cited (including links to both the formal publication through the relevant DOI and the license). See: https://creativecommons.org/licenses/by-nc-nd/4.0/.

\section{References}

1. Ikomi F, Kawai Y, Ohhashi T. Recent advance in lymph dynamic analysis in lymphatics and lymph nodes. Ann Vasc Dis 2012;5:258-68.

2. Tanis PJ, Nieweg OE, Valdés Olmos RA, et al. Anatomy and physiology of lymphatic drainage of the breast from the perspective of sentinel node biopsy. J Am Coll Surg 2001;192:399-409.

3. Hsu MC, Itkin M. Lymphatic anatomy. Tech Vasc Interv Radiol 2016;19:247-54.

4. Prodromidou A, Iavazzo C, Fotiou A, et al. The application of fibrin sealant for the prevention of lymphocele after lymphadenectomy in patients with gynecological malignancies: a systematic review and meta- analysis of randomized controlled trials. Gynecol Oncol 2019;153:201-8.

5. Zikan M, Fischerova D, Pinkavova I, et al. A prospective study examining the incidence of asymptomatic and symptomatic lymphoceles following lymphadenectomy in patients with gynecological cancer. Gynecol Oncol 2015;137:291-8.

6. Nadolski GJ, Chauhan NR, Itkin M. Lymphangiography and lymphatic embolization for the treatment of refractory chylous ascites. Cardiovasc Intervent Radiol 2018;41:415-23.

7. Kim EY, Hwang HS, Lee HY, et al. Anatomic and functional evaluation of central lymphatics with noninvasive magnetic resonance lymphangiography. Medicine (Baltimore) 2016;95:e3109.

8. Yoshida RY, Kariya S, Ha-Kawa S, et al. Lymphoscintigraphy for imaging of the lymphatic flow disorders. Tech Vasc Interv Radiol 2016;19:273-6.

9. Pieper CC, Hur S, Sommer CM, et al. Back to the future: lipiodol in lymphography-from diagnostics to theranostics. Invest Radiol 2019;54:600-15.

10. Rajebi MR, Chaudry G, Padua HM, et al. Intranodal lymphangiography: feasibility and preliminary experience in children. J Vasc Interv Radiol 2011;22:1300-5.

11. Dori Y, Zviman MM, Itkin M. Dynamic contrastenhanced MR lymphangiography: feasibility study in swine. Radiology 2014;273:410-6.

12. Krishnamurthy R, Hernandez A, Kavuk S, et al. Imaging the central conducting lymphatics: initial experience with dynamic MR lymphangiography. Radiology 2015;274:871-8.

13. Weinberger V, Cibula D, Zikan M. Lymphocele: prevalence and management in gynecological malignancies. Expert Rev Anticancer Ther 2014;14:307-17.

14. Kim SW, Hur S, Kim SY, et al. The efficacy of lymph node embolization using n-butyl cyanoacrylate compared to ethanol sclerotherapy in the management of symptomatic lymphorrhea after pelvic surgery. J Vasc Interv Radiol 2019;30:195-202.e1.

15. Bielsa S, Pardina M. Refractory chylothorax: where do we go now? Curr Pulmonol Reports 2019;8:60-7.

16. Bellini C, Cabano R, De Angelis LC, et al. Octreotide for congenital and acquired chylothorax in newborns: a systematic review. J Paediatr Child Health 2018;54:840-7.

17. Mahrer A, Ramchandani P, Trerotola SO, et al. Sclerotherapy in the management of postoperative lymphocele. J Vasc Interv Radiol 2010;21:1050-3.

18. Cope C, Kaiser LR. Management of unremitting 
chylothorax by percutaneous embolization and blockage of retroperitoneal lymphatic vessels in 42 patients. J Vasc Interv Radiol 2002;13:1139-48.

19. Hur S. Facing the truth: penetration of vital organs during thoracic duct embolization. J Vasc Interv Radiol 2020;31:80-1.

20. Kim PH, Tsauo J, Shin JH. Lymphatic interventions for chylothorax: a systematic review and meta-analysis. J Vasc Interv Radiol 2018;29:194-202.e4.

21. Kortes N, Radeleff B, Sommer CM, et al. Therapeutic lymphangiography and CT-guided sclerotherapy for the treatment of refractory lymphatic leakage. J Vasc Interv Radiol 2014;25:127-32.

22. Matsumoto T, Yamagami T, Kato T, et al. The effectiveness of lymphangiography as a treatment method for various chyle leakages. Br J Radiol 2009;82:286-90.

23. Kos S, Haueisen H, Lachmund U, et al. Lymphangiography: forgotten tool or rising star in the diagnosis and therapy of postoperative lymphatic vessel leakage. Cardiovasc Intervent Radiol 2007;30:968-73.

24. Gruber-Rouh T, Naguib NNN, Lehnert T, et al. Direct lymphangiography as treatment option of lymphatic leakage: Indications, outcomes and role in patient's management. Eur J Radiol 2014;83:2167-71.

25. Alejandre-Lafont E, Krompiec C, Rau WS, et al. Effectiveness of therapeutic lymphography on lymphatic leakage. Acta Radiol 2011;52:305-11.

26. Kawasaki R, Sugimoto K, Fujii M, et al. Therapeutic effectiveness of diagnostic lymphangiography for refractory post-operative chylothorax and chylous ascites: correlation with radiologic findings and preceding medical treatment. AJR Am J Roentgenol 2013;201:659-66.

27. Hur S, Shin JH, Lee IJ, et al. Early experience in the management of postoperative lymphatic leakage using lipiodol lymphangiography and adjunctive glue embolization. J Vasc Interv Radiol 2016;27:1177-86.e1.

28. Dinç H, Oğuz Ş, Sarı A. A novel technique in the treatment of retroperitoneal lymphatic leakage: direct percutaneous embolization through the leakage pouch. Diagn Interv Radiol 2015;21:419-22.

29. Ching KC, Santos E, McCluskey K, et al. CT-guided injection of N-butyl cyanoacrylate glue for treatment of chylous leak after aorto-mesenteric bypass. Cardiovasc Intervent Radiol 2014;37:1103-6.

30. Itou C, Koizumi J, Myojin K, et al. A case of refractory chylous ascites after nephrectomy successfully treated with percutaneous obliteration using adhesive glue. Jpn J Radiol 2013;31:71-4.

31. Lee H, Kim SJ, Hur S, et al. The feasibility of mesenteric intranodal lymphangiography: its clinical application for refractory postoperative chylous ascites. J Vasc Interv Radiol 2018;29:1290-2.

32. Baek Y, Won JH, Chang SJ, et al. Lymphatic embolization for the treatment of pelvic lymphoceles: preliminary experience in five patients. J Vasc Interv Radiol 2016;27:1170-6.

33. Chick JF, Reddy SN, Nadolski GJ, et al. Single-session endolymphatic glue embolization of lymphocele after heart transplantation. J Vasc Interv Radiol 2016;27:929-30.
Cite this article as: Hur S, Jun H, Jeong YS. Novel interventional radiological management for lymphatic leakages after gynecologic surgery: lymphangiography and embolization. Gland Surg 2021;10(3):1260-1267. doi: 10.21037/gs-2019ursoc-10 